\title{
Screening, Brief Intervention and Referral to Treatment (SBIRT) training for nurses in acute care settings: Lessons Learned
}

\author{
*Rhonda Schwindt, DNP, RN, PMHNP-BC
}

Assistant Professor of Nursing

Director Psychiatric/Mental Health Nurse Practitioner Program

George Washington University

1919 Pennsylvania Avenue, NW

Washington, DC 20006

rhondaschwindt@gwu.edu

Jon Agley, PhD, MPH

Assistant Research Scientist

IU School of Public Health and the Institute for Research on Addictive Behavior

501 N. Morton St, Suite 110

jagley@indiana.edu

Robin Newhouse, PhD, RN, NEA-BC, FAAN

Dean and Distinguished Professor

Indiana University School of Nursing

600 Barnhill Drive, NU 132 Indianapolis, IN 46202

newhouse@iu.edu

Melora Ferren, MSN, RN-BC

Executive Director to the Executive Vice President and the Chief Nurse Executive Indiana University Health

Fairbanks Hall, 340 West 10th St. Indianapolis, IN 46202

mferren@iuhealth.org

\section{*Corresponding author}

Funding source: Indiana University Health (US) (VFGC-020)

Indiana Clinical and Translational Sciences Institute (US); National Institutes of Health, National Center for Advancing Translational Sciences, Clinical and Translational Sciences Award (UL1TR001108)

This is the author's manuscript of the article published in final edited form as:

Schwindt, R., Agley, J., Newhouse, R., \& Ferren, M. (2019). Screening, brief intervention and referral to treatment (SBIRT) training for nurses in acute care settings: Lessons learned. Applied Nursing Research, 48, 19-21. 


\section{INTRODUCTION}

Behavioral health and substance use disorders (SUDs) represent a substantial portion of global disease burden and are the leading causes of years lived with disability across the world (Whiteford et al., 2015). In the United States, tobacco and alcohol use are associated with leading causes of morbidity and mortality (Johnson et al., 2014) and fatal drug overdoses (excluding alcohol) are a significant contributor to injury deaths (Ruhm, 2018). These data highlight the importance of developing sustainable models of care and the necessary workforce to implement those models across healthcare systems. Screening, brief intervention, and referral to treatment (SBIRT) is an evidence-based practice framework to enable the identification of atrisk substance users (Babor et al., 2007) that offers a streamlined protocol for integrating substance use prevention and treatment into routine medical care (McCance-Katz \& Satterfield, 2012). SBIRT includes three primary components: 1) Screening for risk using one or more validated instruments; 2) Brief intervention (motivational counseling) for patients with moderate risk; and 3) Referral to treatment for patients with severe risk or probable dependence (Substance Abuse and Mental Health Services Administration, n.d.).

Nurses are well-equipped and positioned to deliver SBIRT interventions. While training strategies have been developed and are feasible across a variety of settings (Mitchell et al., 2017; Cook et al., 2018), several challenges remain for SBIRT in nursing, including inadequate training and knowledge, an absence of implementation and workflow protocols, concerns about the time needed to deliver interventions, a lack of common data elements in the electronic medical record (EMR), role ambiguity, and reimbursement policies (Wamsley et al., 2018). As part of a waitlist randomized cluster trial for implementation of SBIRT in a large Midwestern 
hospital system, our team trained one registered nurse $(\mathrm{RN})$ from each adult acute care hospital $(N=14)$ using a train-the-trainer approach (Newhouse et. al, 2018) and collected post-training feedback. The purpose of this research brief is to share evaluation data and site coordinators' feedback to advance understanding of how best to structure SBIRT train-the-trainer events for nurses, especially those in managerial roles.

\section{METHODS}

The Conceptual Model for Considering the Determinants of Diffusion, Dissemination and Implementation of Innovations in Health Services Delivery and Organization was used as a framework for this study (Greenhalgh, Robert, Macfarlane, Bate, \& Kyriakidou, 2004). This model offers a guide for the adoption and implementation of innovations within organizations based on a narrative synthesis of theory and research. It posits that interventions that are based on assessment of barriers, use multiple strategies, and are system focused are more likely to be effective.

Chief Nursing Officers of each participating hospital selected one RN as a study site coordinator who was best positioned to lead study activities at their organization. Individual characteristics of site coordinators was not collected. The post-training evaluation was completed to solicit feedback about the training approach and learning outcomes and was not intended to link characteristics to evaluation data or SBIRT implementation.

\section{Figure 1. Training Content}
a. Study Overview (45 mins)
b. Substance Use Overview (45 mins)
c. Introduction to SBIRT (45 mins)
d. Review of Screening for SUDs (30 mins)
e. Conceptual Overview of MI (60 mins)
f. Guidelines for BI and Referral (90 mins)
g. Systems Issues for SBIRT Implementation (30 mins)
h. SBIRT Competency Testing and Review (60 mins)
i. SBIRT Site Implementation (30 mins) j. Question and Answer Period (45 mins)
${ }^{*} M I=$ Motivational Interviewing; $S U D=$ Substance Use Disorder $; B I=$ Brief Intervention 
Twelve of the fourteen site coordinators attended one of two face-to-face training sessions $(n=6$ intervention, $n=6$ waitlist). Sessions were separated by six months because the waitlist protocol called for an equivalent but staggered SBIRT implementation process for two randomly-selected clusters of seven hospitals. Trainings were led by the same interdisciplinary team composed of two doctorate-prepared university nursing faculty, one doctorate-prepared health behavior and SBIRT specialist, the Program Manager for Pain Services and Chemical Dependence from a participating system hospital (not from a study unit), and the Executive Director of Discovery and Contemporary Nursing Practice within the healthcare system. Both the waitlist and intervention group received the same one-day, 8-hour training in their respective sessions (Figure 1). The two site coordinators who were not able to attend a face-to-face training completed an online training module and received 1:1 instruction from a study team member.

Principles of adult learning theory were used to guide the development and implementation of the training. Training activities were focused on the SBIRT process and implementation strategies (e.g., use of screening tools, motivational interviewing). Site coordinators were encouraged to discuss specific barriers to implementation related to their respective organizations, and strategies to overcome the identified barriers. In addition, training team members actively engaged participants in role play, skills validation, self-reflection and individualized feedback to promote knowledge and skill retention (Lane et al., 2008). Prior to attending the training, all site coordinators completed a baseline assessment of their hospital for structural capacity (e.g., stakeholder buy-in), staff capacity (e.g., staff competencies), organizational support (e.g., leadership support), technical capabilities (e.g., access to technology), and fiscal capacity (e.g. locked cabinet for study materials). 
Post-training evaluation data were collected at the end of each session from site coordinators who attended the face-to-face training session using a 22-item questionnaire designed by the principal investigator. Site coordinators indicted the degree to which they agreed with the overall quality of the training ( $n=7$ questions) on a 5-point Likert scale from 1 (strongly agree) to 5 (strongly disagree), and their satisfaction with the training materials, facilities, and presenters ( $n$ = 12 questions ) ranging from 1 (extremely satisfied) to 5 (extremely dissatisfied). Three openended questions were included to solicit additional comments. Descriptive statistics and frequencies were assessed, and qualitative data summarized.

\section{RESULTS}

\section{Descriptive}

The majority of site coordinators strongly agreed that the training objectives were met $(75 \%)$, information was presented in a logical manner $(67 \%)$, training prepared participants to implement SBIRT at their own facility (58\%), and the training facilitated knowledge and ideasharing among participants (100\%). In addition, site coordinators were extremely satisfied with the presentation content (83\%) and length of the training $(50 \%)$.

\section{Qualitative}

Site coordinators provided consistent responses when asked what topics were most beneficial, emphasizing motivational interviewing (MI) and introduction to SBIRT. One respondent identified the "tools and resources available to site coordinators" as being especially helpful. In response to the prompt, "what subjects would you have liked to have more content on," replies varied widely and reflected a level of discomfort with their supervisory capacity within the planned SBIRT implementation program. While two individuals identified MI/brief intervention 
as the most important topic of interest, other responses focused on implementation protocols including, change readiness in relation to teaching staff to implement SBIRT, interacting with, and readiness of, patients, a need to understand the current process before implementation, burden of planning, the expectation of participating in synchronous meetings and site visits "on top of actual work hours," and the methods needed for implementation. One site coordinator highlighted general concerns about SBIRT implementation stating, "training was very informative, but I'm scared about not being able to implement it correctly."

\section{DISCUSSION}

Site coordinators' positive opinions of the SBIRT training were consistent with numerous published studies focused on nursing students as trainees (Mitchell et al., 2013; Gotham et al., 2015; Knopf-Amelung et al., 2018). The curricular aspects, described in Figure 1, appeared to match coordinators' expectations, and the order in which they were taught was perceived as logical. The general content and length were also seen as acceptable. Although $58 \%$ of site coordinators agreed they were ready to implement SBIRT after the training, others were hesitant, and several coordinators qualitatively expressed concern regarding their ability to support SBIRT implementation. This is not surprising given the training challenges identified in prior research (Wamsley et al., 2018). At the same time, the site coordinators were provided with an atypically high amount of systematic and implementation-related content within the training (e.g., versus clinically-focused content), even compared to robust, strategically-developed SBIRT curricula for nurses (Broyles, Kraemer, Kengor, \& Gordon, 2013). We included this content to support site coordinators' supervisory needs within the larger research project, however, an SBIRT train-thetrain approach may need even more preparatory implementation education. In addition, site coordinators' concerns about SBIRT implementation lend credence to the need for formalized 
partnerships between site coordinators and nursing leadership to ensure evidence-based implementation at the unit level.

The evaluation data must be interpreted within the context of several limitations. First, the data were collected to inform future SBIRT implementation projects and were not intended for generalizable conclusions. Second, the study sample of trainees was small, and the evaluation of training was not powered for statistical analysis related to training components or trainee characteristics. Finally, the site coordinators were selected because of their demonstrated leadership in clinical and quality initiatives and their ability to successfully lead their unit in SBIRT implementation. As a result, they may have had a higher degree of readiness to serve as a site coordinator.

Evaluation data yielded important information for future similar trainings. While a standardized training approach for SBIRT implementation is recommended and sufficient for baseline training, multiple training methods (e.g., in-person site visits, training materials designed specifically for the unit setting, monthly calls and occasional synchronous or in-person booster sessions) are needed to enhance implementation efforts. An important next step might be a qualitative assessment to test specific training features and methods that work best when implementing new clinical practices using a comparative design. The results of such as study would lend support to unit/organizational implementation of SBIRT. 


\section{References}

Babor, T.F., McRee, B.G., Kassebaum, P.A., Grimaldi, P.L., Ahmed, K., \& Bray, J. (2007). Screening, Brief Intervention, and Referral to Treatment (SBIRT): Toward a public health approach to the management of substance abuse. Substance Abuse, 28(3), 7-30.

Broyles, L.M., Kraemer, K.L., Kengor, C., \& Gordon, A.J. (2013). A tailored curriculum of alcohol screening, brief intervention, and referral to treatment (SBIRT) for nurses in inpatient settings. Journal of Addictions Nursing, 24(3), 130-141.

Cook, P.F., Aagaard, L., Bowler, F., Rosenthal, L., Avery, L.K., \& Weber, M. (2018). Screening, brief intervention, and referral to treatment: Nurses Helping Colorado training program. Journal of Nursing Education, 57(8), 476-482.

Gotham, H.J., Knopf-Amelung, S., Krom, L., Stilen, P., \& Kohnle, K. Competency-based SBIRT training for health-care professionals: Nursing and social work students. Addiction Science \& Clinical Practice, 10(S1), A14.

Greenhalgh, T., Robert, G., Macfarlane, F., Bate P., \& Kyriakidou O. (2004). Diffusion of innovations in service organizations: systematic review and recommendations. Milbank Quarterly, 82(4) 581-629.

Johnson, N.B., Hayes, L.D., Brown, K., Hoo, E.C., Ethier, K.A., et al. (2014). CDC National Health Report: Leading causes of morbidity and mortality and associated behavioral risk and protective factors - United States, 2005 - 2013. Morbidity and Mortality Weekly Report, 63(4), 3-27.

Knopf-Amelung, S., Gotham, H., Kuofie, A., Young, P., Stinson, R.M., Lynn, J., et al. (2018). Comparison of instructional methods for screening, brief intervention, and referral to treatment for substance use in nursing education. Nurse Educator, 43(3), 123-127. 
Lane, C., Hood, K., \& Rollnick, S. (2008). Teaching motivational interviewing: Using role play is as effective as using simulated patients. Medical Education, 42(6), 637-644.

McCance-Katz, E.F. \& Satterfield, J. (2012). SBIRT: A key to integrate prevention and treatment of substance abuse in primary care. American Journal of Addiction, 21(2), 176177.

Mitchell, A.M., Kane, I., Lindsay, D.L., Hagle, H., Puskar, K., Aiello, J., et al. (2017). Educating emergency department registered nurses (EDRNs) in screening, brief intervention, and referral to treatment (SBIRT): Changes in attitudes and knowledge over time. International Emergency Nursing, 33, 32-36.

Mitchell, A.M., Puskar, K., Hagle, H., Gotham, H.J., Talcott, K.S., Terhorst, L., et al. (2013). Screening, brief intervention, and referral to treatment: Overview of and student satisfaction with an undergraduate addiction training program for nurses. Journal of Psychosocial Nursing and Mental Health Services, 51(10), 29-37.

Newhouse, R. P., Janney, M., Gilbert, A., Agley, J., Bakoyannis, G., Ferren, M., Mullins, C. Daniel, Johantgen, M., Schwindt, R., Thoele, K. (2018). Study Protocol Testing Toolkit versus Usual Care for Implementation of Screening, Brief Intervention, Referral to Treatment in Hospitals: A Phased Cluster Randomized Approach. Addiction Science \& Clinical Practice, 13:28. https://doi.org/10.1186/s13722-018-0130-4

Ruhm, C.J. (2018). Corrected US opioid-involved drug poisoning deaths and mortality rates, 1999-2015. Addiction, 113(7), 1339-1344.

Substance Abuse and Mental Health Services Administration(n.d). Retrieved from: http://www.samhsa.gov/sbirt/about). 
Wamsley, M., Satterfield, J.M., Curtis, A., Lundgren, L., \& Satre, D.D. (2018) Alcohol and drug screening, brief intervention, and referral to treatment (SBIRT) training and implementation: Perspectives from 4 health professions. Journal of Addiction Medicine, 12(4), 262-72.

Whiteford, H.A., Ferrari, A.J., Degenhardt, L., Feigin, V., \& Vos, T. (2015). The global burden of mental, neurological, and substance use disorders: An analysis from the Global Burden of Disease Study 2010. PLOS One, 10(2), e0116820. 


\section{HIGHLIGHTS}

1. Site Coordinators who attended an one day, 8-hour, training endorsed the value of training in screening, brief intervention, and referral to treatment (SBIRT).

2. Training nurses with supervisory responsibilities may require more preparatory implementation education pre-training.

3. A standardized train-the-trainer approach for SBIRT implementation is sufficient for baseline training, however multiple training methods are needed to enhance implementation methods. 